\title{
Encapsulation and In Vitro Evaluation of Low-Density Lipoprotein with Cholesterol Conjugated Anti-HSP27 and HER2 Proteins as Drug Delivery Enhancement in Ovarian Cancer
}

\author{
Laila Abdulmohsen Jaragh Alhadad* \\ Department of Chemistry, College of Science, Kuwait University, Kuwait
}

*Corresponding author: Laila Abdulmohsen Jaragh Alhadad, Department of Chemistry, College of Science, Kuwait

University, Kuwait

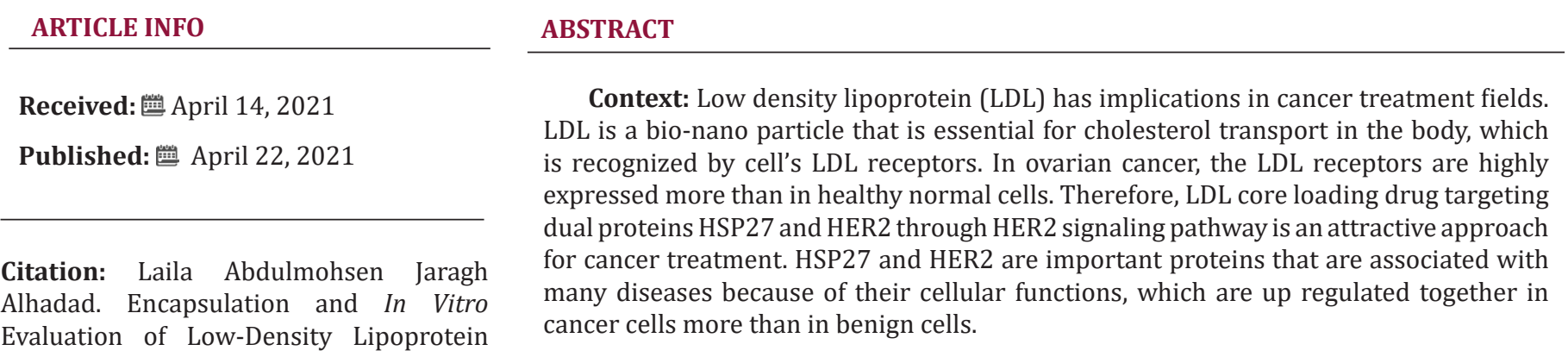

Objective: The present study was designed to target and evaluate cholesterol with Cholesterol Conjugated Anti-HSP27 and HER2 Proteins as Drug Delivery Enhancement in Ovarian Cancer. Biomed J Sci \& Tech Res 35(2)-2021. BJSTR. MS.ID.005675.

Keywords: LDL; Cholesterol Conjugates; Drug Delivery; HSP27; HER2; SKOV3

Abbreviations: LDL: Low Density Lipoprotein; VLDL: Very Low Density Lipoprotein; IDL: Intermediate Density Lipoprotein; HDL: High Density Lipoprotein; DLS: Dynamic Light Scattering conjugated HSP27 and HER2 inhibitor loaded into LDL particles to take the advantages of the metabolic pathways of lipid biochemistry.

Materials and Methods: Previous synthesized cholesterol conjugates loaded into commercial LDL core, simply by mixing, sonicating (1:5 ratio) respectively, followed by particles' size measurements. Then, SKOV3 cells in-vitro growth evaluation using both western blot and MTT assays.

Key Finding: Particles' size was significantly increased after encapsulation of the (cholesterol/Agent 1\&2) into LDL particles. Both cholesterol/Agent 1 and cholesterol/ Agent 2 did not show any precipitation in aqueous solution at both $100 \mu \mathrm{M}$ and $500 \mu \mathrm{M}$. High ovarian cancer cell's (SKOV3) growth inhibition was shown by western blot assay and MTT assay.

Conclusion: LDL encapsulation with cholesterol conjugated anticancer agents proved drug delivery strategy targeting dual proteins which is mimicking the native components of LDL and its metabolic pathway.

\section{Introduction}

LDL is a vehicle that moves, transports and supply both free and esterified cholesterol through the blood stream to different parts of the body [1]. Lipoproteins are endogenous nanoparticles, structurally consisting of a hydrophobic core that contains cholesteryl esters and triacylglycerol, and the hydrophilic shell contains phospholipids and free cholesterol with one apolipoprotein B-100 for receptor recognition [2]. The particle size of lipoproteins

ranges from 10 to $1200 \mathrm{~nm}$, and the size of each type of lipoprotein is as follows: chylomicron, 75-1200 nm; very low density lipoprotein (VLDL), 30-80 nm; intermediate density lipoprotein (IDL), 25-35 $\mathrm{nm}$; low density lipoprotein (LDL), 18-25 nm; and high density lipoprotein (HDL), 8-12 $\mathrm{nm}$. The liver synthesizes and secretes VLDL containing cholesterol ester, which is converted to LDL by lipoprotein lipase enzyme [3,4]. LDL life cycle starts with carrying 
cholesterol and binding to specific LDL cell's receptors through ApoB-100. LDL receptors sit on the outer surface of many types of cells, especially ovarian cells, where they pick up LDLs containing cholesterol circulating from the bloodstream and transport them inside the cell.

Then, LDL becomes internalized and undergoes the endocytosis process. First, LDL fuses through clathrin coated vesicle, and the receptor will be recycled by the endosome $[5,6]$. The endosome breaks down the ligand and then the lysosome, the hydraulic enzyme releases and provides the cell with cholesterol cargo which is either used or stored by the cell. The extra cholesterol from the cells is brought back to the liver by high density lipoprotein (HDL) in a process known as reverse cholesterol transport $[7,8]$ as shown in (Figure 1). It has been found that the LDL receptor is over-expressed in numerous cancers more than in normal tissues [9-12]. Various lipid metabolic pathways, especially those involving fatty acid biosynthesis, along with phospholipids and their enzyme systems, have been found to be involved specifically in ovarian cancer. Ovarian cells under normal physiological conditions rely on the use of endogenously synthesized cholesterol, which is delivered by low density lipoprotein (LDL), but in case of ovarian cancer, cholesterol uptake will be more pronounced because the cells need it for new cell membrane synthesis [13-17].

These findings have led many researchers to investigate the possibility of exploring LDL as a delivery vehicle for cancer diagnostics and therapeutics. Awwad, etal. [18] stated that targeting cancer using cholesterol conjugates to be taken by the natural LDL is considered a perfect drug delivery system for cancer treatment and clinical diagnosis [18-21]. There are three different ways in which diagnostic or therapeutic agents can be incorporated into LDL. First, drugs can be loaded into LDL via a covalent attachment to the amino acid residues of apolipoprotein Apo-B100 (protein loading). Second, drugs can be loaded through intercalation into the phospholipid's monolayer of LDL (surface loading). Third, drugs can be loaded via substitution of agents into the lipid core of LDL (core loading) [22-24]. In general, conjugation of cholesterol to an active agent targets dual proteins HSP27 and HER2 through HER2 pathway, encapsulated into LDL is an attractive approach for cancer therapy (Figure 1).

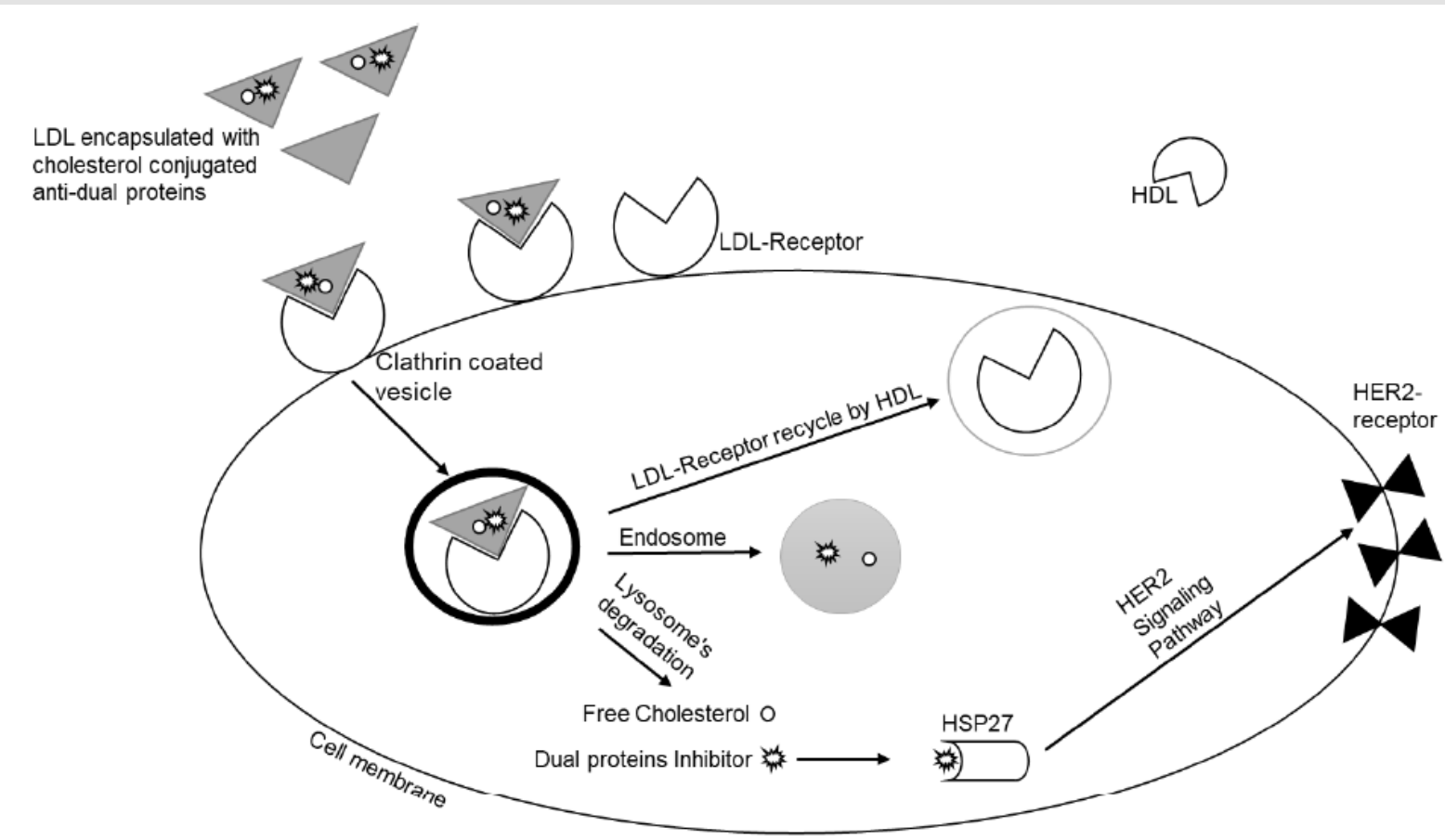

Figure 1: Schematic representation of LDL life cycle and cholesterol metabolism. Releasing cholesterol conjugates inside the cell to target HSP27 then reduces HER2 expression through HER2 pathway.

The dual strategies conjugation and encapsulation enhance the ability of the agent to reach and accumulate in the middle of cancer cells without being recognized by the cell's defenses mimicking the endogenous LDL [25-28]. Therefore, our goal in this present study was an effort to combine two strategies conjugation and encapsulation to target ovarian cancer cells (SKOV3 cell line that is highly expresses HSP27 protein) in preclinical models and to benefit from the lipid metabolism. First, cholesterol conjugated HSP27 and HER2 inhibitor is previously synthesized and characterized [29,30] as an anti-cancer drug. Secondly, loading cholesterol conjugates into commercially available LDL. Agent 1 and agent 2 (Figures 2 \& 3) first target HSP27 function followed by reduction of HER2 expression through HER2 signaling pathway which can be used as an effective multi- targeted strategy for cancer therapy. 


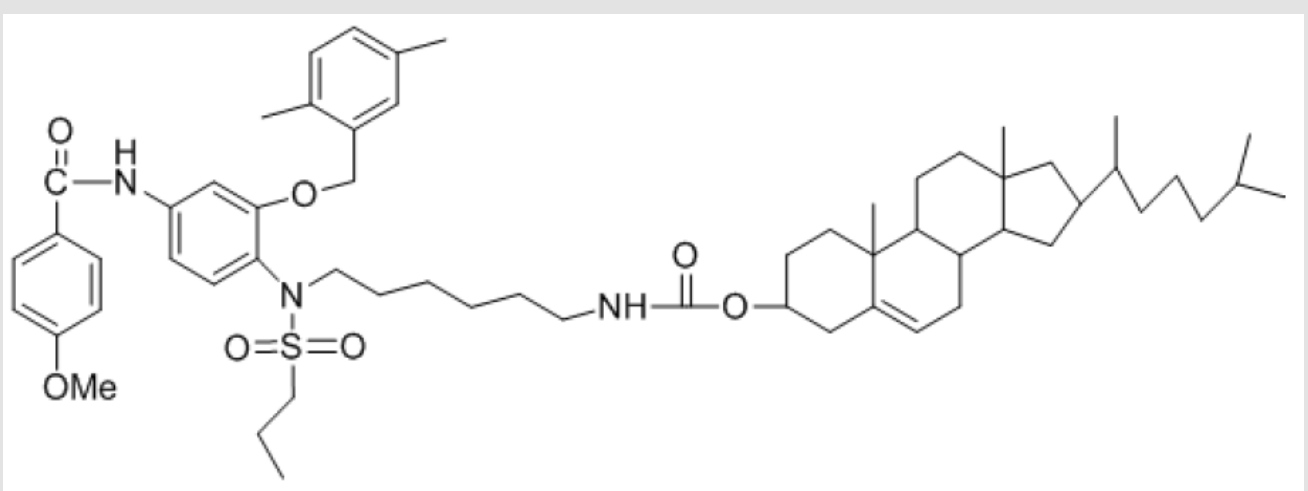

Figure 2: Molecular structures of cholesterol conjugated Agent 1 targeting HSP27 and HER2.

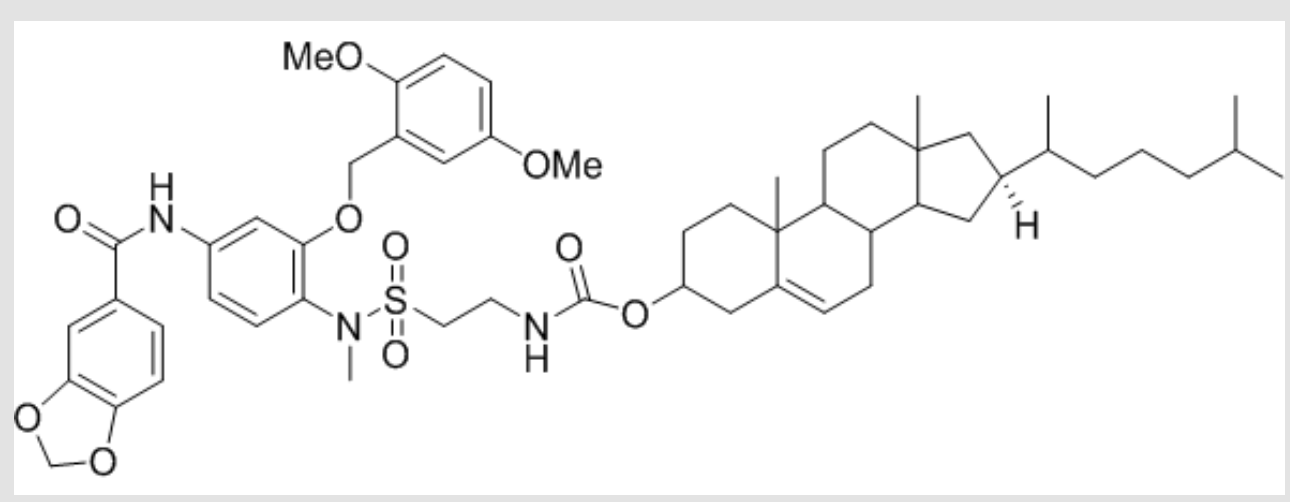

Figure 3: Molecular structures of cholesterol conjugated Agent 2 targeting HSP27 and HER2.

\section{Materials and Methods}

\section{Materials}

Human ovarian cancer cell line SKOV3 was obtained from American Type Culture Collection (ATCC). Cholesterol conjugated dual proteins inhibitors were previously synthesized in our lab. Cell culture's media and other supplements such as 3-(4, 5-dimethylthiazol-2-yl)-2, 5-diphenyl-2 tetrazolium bromide (MTT) were obtained from Sigma-Aldrich (Milwaukee, WI). Chemicals and reagents are commercially available and ready for direct use without need for any preparation. Biological investigation experiment was done at Kuwait University.

\section{Methods}

Western Blot Assay: SKOV3 confluent ovarian cancer cells' dishes were washed with PBS collected, and harvested by a scraper. This action was followed by the re-suspension of the cells with PBS and then centrifuging the collected sample at $3500 \mathrm{~g}$. The supernant liquid was then removed, and the collected cell pellets were lysed by mixing them with RIPA, PI, and EDTA. The proteins were vortexed and centrifuged for one hour at $16500 \mathrm{~g}$. The proteins were boiled with $2 x$ loading buffer for five minutes and then normalized and electrophoresed on a $10 \%$ SDS-polyacrylamide gel. The gel contains the proteins that were transferred to a nitrocellulose membrane followed by blocking of the nonspecific proteins with 5\% NFDM in PBST. The membranes were rinsed three times for five minutes with PBST $(0.1 \%$ Tween 20$)$. Then, the membrane was incubated with the primary antibody (rabbit 1:1000 dilution) and secondary antibody (HRP 1:1000 dilution) that targets HER2, for one hour followed by washing steps with PBST. Similar steps were repeated for the membrane incubated with HSP27 (rabbit 1:500 dilution) and then the membrane was prepared for the visualization of the bands by chemiluminescence. B-actin (1:1000 dilutions) was used as a control.

Dynamic Light Scattering: Commercial LDL was obtained for Sigma Louis and used to encapsulate cholesterol conjugate at ratio (5:1) respectively. The particles size was measured using dynamic light scattering (DLS) technique. First, pure LDL was mixed with PBS and the particles size was measured. Second, LDL encapsulated cholesterol conjugated inhibitor was prepared as following, ten $\mu \mathrm{l}$ of pure LDL and LDL encapsulated cholesterol conjugated inhibitors were added into $1000 \mu \mathrm{l}$ PBS and the sample particles size diameters were measured by Brookhaven software, pure PBS was used as a blank.

Cell Culture and MTT Assay: The cells were maintained in RPMI1640 medium containing 10\% FBS and $10 \mathrm{~mL}$ penicillinstreptomycin and $100 \mu \mathrm{l}$ ciprofloxacin. Cell cultures were grown at $37{ }^{\circ} \mathrm{C}$ in a humidified atmosphere of 5\% CO2 incubator. MTT 
assay was performed by monitoring the reduction of yellow MTT to a colored product. After culturing the cells in the 2D monolayer cell culture, cells were harvested and then seeded with RPMI1640 medium into 96-well and incubated overnight. Then, cells were treated with different concentrations of anticancer agents (cholesterol/Agent 1 and cholesterol/Agent 2) in four replicates each and incubated for $72 \mathrm{hr}$. DMSO/LDL control wells received concentrations equal to those in the drug-treated cell wells. The cell viability was determined by MTT reagent $100 \mu \mathrm{l}$ of $0.5 \mathrm{mg} / \mathrm{ml}$ in fresh medium, added after the removal of the old medium, and the cells were incubated for one hour. Supernatants were removed from the wells, and then $100 \mu \mathrm{l}$ DMSO was added on the reduced MTT dye. The final absorbency measurement was determined using a plate reader at $570 \mathrm{~nm}$ and the values were normalized to controls.

\section{Statistical Analysis}

Graph Pad Prism software (Graph Pad Software Incorporated) and Microsoft Excel (Microsoft Corporation) were used to determine the statistical and graphical information. IC $_{50}$ values were normalized using nonlinear regression analysis. The experiments were done quadruplicated and repeated at least three times.

\section{Results and Discussion}

\section{SKOV3 Examination for Drug Potency Using Western Blot Assay Targeting HSP27}

In the previous research studies, cholesterol conjugated dual proteins inhibitor was successfully prepared and characterized $[29,30]$. The potent cholesterol/agents 1 and 2 were chosen for further tests to prove their potency to inhibit the growth of SKOV3 cells. The cell lysates were collected, and we ran a gel electrophoreses experiment to obtain the bands. The data revealed that both agents were potent in targeting HSP27 function and reduce HER2 expression through HER2 pathway based on DMSO control (Figure 4).

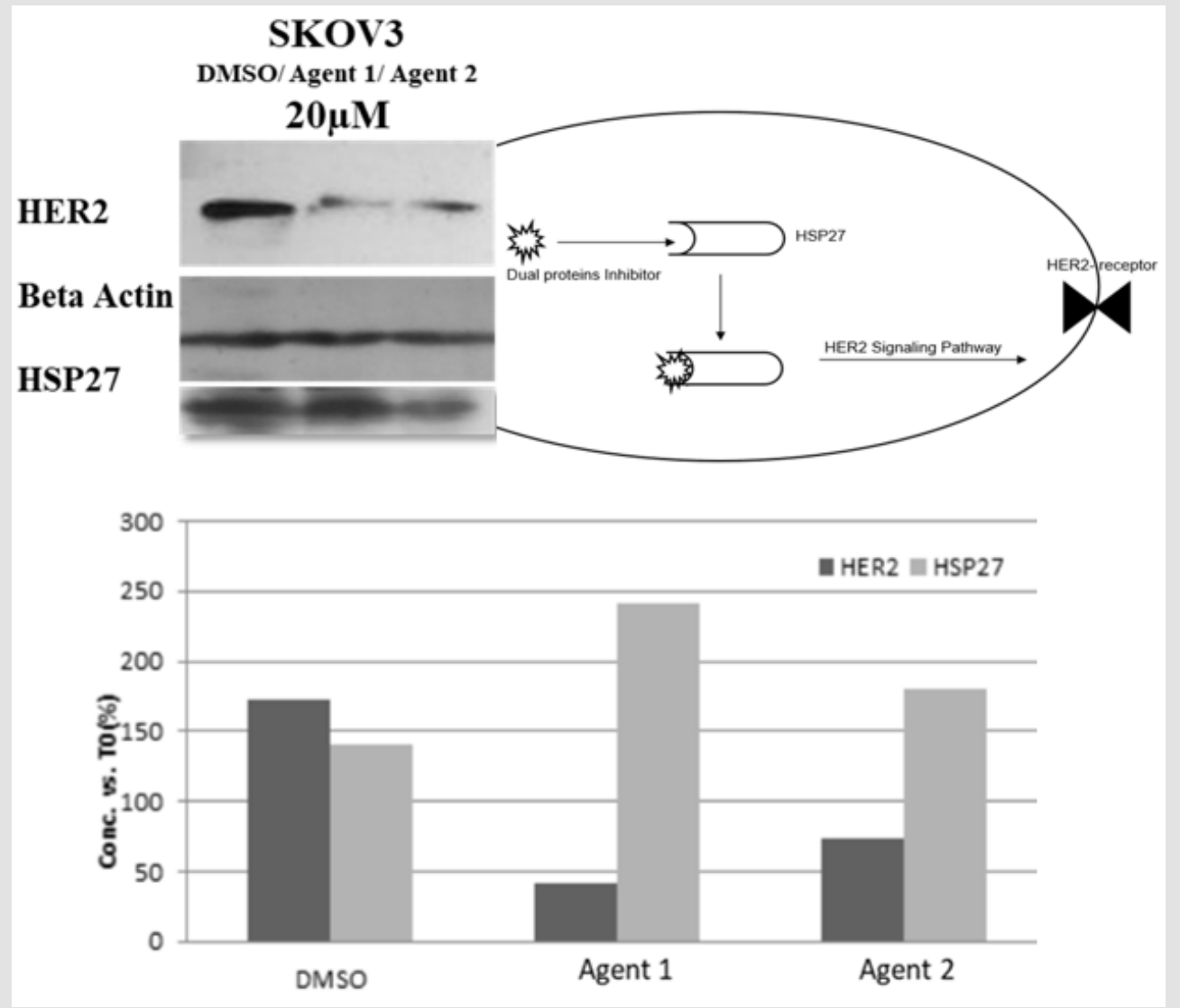

Figure 4: Western blot assay showed that agents 1 and 2 able to target HSP27 function in SKOV3 cells and reduce HER2 expression through HER2 pathway.

Loading Cholesterol Conjugates into LDL Particles; Particle Size Measurements Using DLS Technique

Cholesterol/Agents 1 and 2 both were loaded into a commercially available LDL. First, pure LDL particle size measured and had a diameter about $48.6 \mathrm{~nm}$ with simple hand mixing. On the other hand, sample of pure LDL particles mixed and sonicated, its size reached up to $78.1 \mathrm{~nm}$. Second, after loading cholesterol/ Agent 1 agent into LDL particles mixed only by hand, its particle size was $196.6 \mathrm{~nm}$, while when the same agent mixed, sonicated, 
and left for $24 \mathrm{hr}$. to rest, the LDL particle size became $249.6 \mathrm{~nm}$. Same steps repeated for cholesterol/Agent 2 and the measured particle size of simply mixed sample by hand, was $410.7 \mathrm{~nm}$ but sample mixed, sonicated, and left overnight its particle size became $591 \mathrm{~nm}$. Generally, we found that the particle sizes for the mixture of cholesterol conjugates in LDL particles without sonication had small diameters compared to the sonicated samples. This is because after sonication step, LDL particles broken down and to allow the cholesterol/anti-cancer agents to enter it while reforming overnight as shown in (Table 1) \& (Figure 5).
(A)

(B)

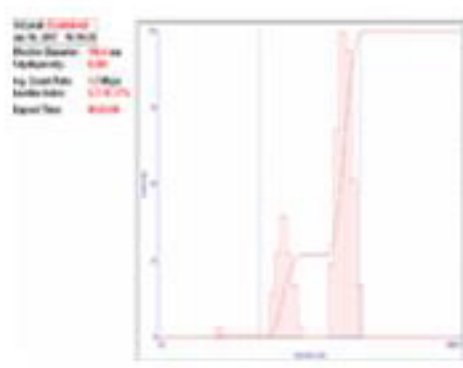

(C)

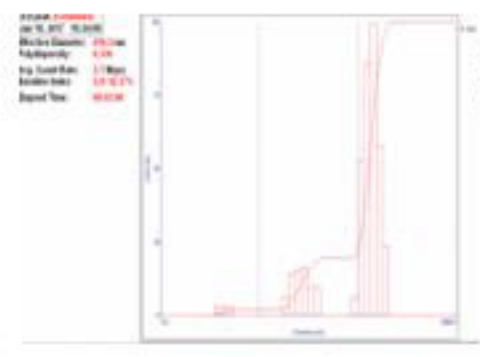

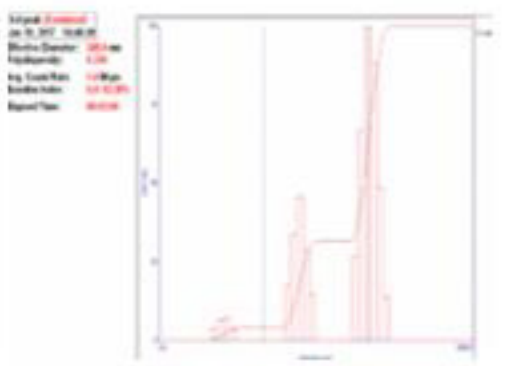
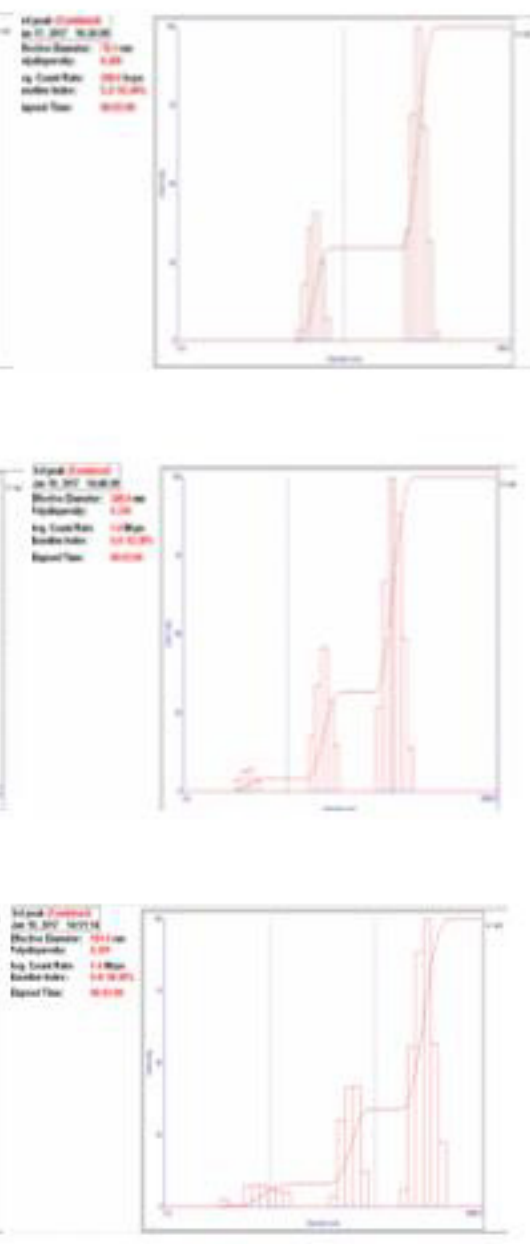

Figure 5: LDL particle size was measured by DLS technique in PBS.

(A) Free LDL particles,

(B) LDL mixed/sonicated with cholesterol conjugated agent 1 ,

(C) LDL mixed/sonicated with cholesterol conjugated agent 2.

Sonicated samples have larger diameter more than non-sonicated, which proofed the encapsulation.

Table 1.

\begin{tabular}{|l|c|c|}
\hline \multicolumn{1}{|c|}{ Particles } & Diameter of Non-Sonicated Sample $\mathbf{n m} \pm$ SD & Diameter of Sonicated Sample $\mathbf{n m} \pm \mathbf{S D}$ \\
\hline (A) LDL & $48.60 \pm 0.08$ & $78.1 \pm 0.650$ \\
\hline (B) Agent 1+cholesterol +LDL & $196.6 \pm 5.97$ & $249.6 \pm 12.5$ \\
\hline (C) Agent 2+cholesterol +LDL & $410.5 \pm 17.7$ & $591.0 \pm 13.1$ \\
\hline
\end{tabular}

The proof that cholesterol conjugates loaded into LDL particles is the solubility of the conjugates in the aqueous solution. Both Cholesterol/Agent 1 and cholesterol/Agent 2 precipitated when treated with SKOV3 cells in the aqueous solution at $25 \mu \mathrm{M}$. However, when SKOV3 cells treated with Cholesterol/Agent 1 and
cholesterol/Agent 2 both loaded into LDL particles, both did not show any precipitation in aqueous solution at both $100 \mu \mathrm{M}$ and $500 \mu \mathrm{M}$, which is the direct evidence that Cholesterol/Agent 1 and cholesterol/Agent 2 were loaded into LDL particles. 


\section{The Cytotoxicity Effects of the Potent Cholesterol Conjugates Encapsulated into LDL Treated with Ovarian Cancer Cells}

MTT assay data summarized in (Table 2) \& (Figure 6). SKOV3 cells treated with both agents mixed with LDL particles simply mixed by hand did not show any cell growth inhibition. This result was expected because the Cholesterol/Agents 1 and 2 did not enter the LDL particles yet. Therefore, Cholesterol/Agents 1 and 2 loaded into LDL via both mixing and sonication, and then treated with SKOV3 cells, the $\mathrm{IC}_{50}$ values are still very high, and the agents did not show any growth inhibition assuming that this is due the competition between the media's LDL and our LDL containing cholesterol/anti-cancer agent. Therefore, SKOV3 cells treated with the Cholesterol/Agents 1 and 2 in media containing FBS depleted LDL. Surprisingly, the LDL containing Cholesterol/Agents 1 and 2, IC $_{50}$ values were improved and had the strength to inhibit SKOV3 cell growth at $36 \mu \mathrm{M}$. This indicated that LDL containing Cholesterol/ Agents 1 and 2 inhibitors entered the cell through LDL receptor via endocytosis process then targets both proteins HSP27 and then reduced HER2 expression through HER2 pathway.

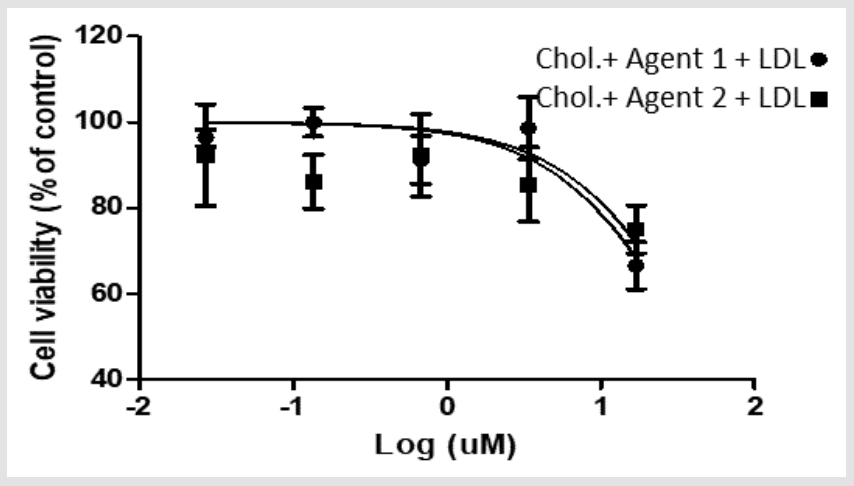

Figure 6.

Table 2: Cytotoxicity profile of SKOV3 cell growth inhibition's, IC $_{50}$ values treated with LDL containing cholesterol conjugates: (A) Simple mixing; (B) Sonication \& (C) Treated with depleted LDL.

\begin{tabular}{|c|c|c|c|}
\hline \multirow{2}{*}{ Compound } & \multicolumn{3}{|c|}{ IC $_{50}$ Values to Inhibit SKOV3 Cell Growth $\mu$ M \pm SD } \\
\cline { 2 - 4 } & (A) & (B) & (C) \\
\hline Agent 1 + Cholesterol/LDL & $>100$ & $>100$ & $36.2 \pm 13.7$ \\
\hline Agent 2 + Cholesterol/LDL & $>100$ & $>100$ & $36.8 \pm 10.4$ \\
\hline
\end{tabular}

DMSO/LDL was used as a control.

Generally, tests were done quadruplicated and repeated at least three times for each sample. The control used was DMSO/LDL. Previous research studies reported that conjugation of anticancer agents to cholesterol is perfect strategy to reduce cancer cell growth $[18,30]$. Loading this conjugate into LDL mimics the native LDL by cholesterol transport and LDL- receptor uptake and accumulation in the middle of cancer cells. Therefore, this research was designed to encapsulate committal available LDL with cholesterol conjugated to anticancer agents targeting dual proteins HSP27 and HER2 through HER2 pathway. The current research data confirmed that LDL containing cholesterol conjugates have the potency to inhibit SKOV3 cell growth by binding the LDL to the LDL-receptor and enters the cell and leave the cargo in the middle. In sum, the development of new medicinal compounds and combination of targeting strategies for cancer treatment has become one of the

\section{Conclusion}

In this study, drug-lipid conjugates have been encapsulated into LDL to take the advantages of the metabolic pathways of lipid biochemistry, improve drug delivery to cancer cells, increase target specificity and increase cellular uptake. The combination of targeting strategies using cholesterol conjugates agent 1 and 2 and LDL particles as a carrier improves drug delivery and accumulation in ovarian cancer SKOV3 cells, which mimics the endogenous LDL containing cholesterol esters, life cycle and metabolism. Our data confirmed that LDL is the vehicle for cholesterol that entered ovarian cancer SKOV3 cells through LDL receptor via endocytosis process. In addition, LDL containing cholesterol conjugates showed SKOV3 cell growth inhibition. This study is an attractive strategy for drug delivery. 


\section{Declaration of Interest}

The author reports no conflict of interest to declare. The author alone is responsible for the contact and writing of this article.

\section{Funding}

This study was supported by grant (SC14/18) from the research sector at Kuwait University.

\section{Acknowledgement}

Author expresses her sincere gratitude toward Research Facilities at Kuwait University (RSPU).

\section{References}

1. Chen Y, Liu G, Guo L, Wang H, Fu Y, et al. (2015) Enhancement of tumor uptake and therapeutic efficacy of EGFR-targeted antibody cetuximab and antibody-drug conjugates by cholesterol sequestration. Int J Cancer 136(1): 182-94.

2. Alanazi F, Haq N, Radwan A, Alsarra IA, Shakeel F (2015) Formulation and evaluation of cholesterol-rich nano emulsion (LDE) for drug delivery potential of cholesteryl-maleoyl-5-fluorouracil. Pharm Dev Technol 20(3): 266-270.

3. Alexandre A, Jesus P, Silvia R, Amancio RF, Souen JS, et al. (2001) Uptake of a Cholesterol-Rich Emulsion by Neoplastic Ovarian Tissues. Gynecologic Oncology 82(1): 84-87.

4. Joseph L, Michael S (2009) The LDL Receptor. Arterioscler Thromb Vasc Biol 29(4): 431-438.

5. Anderson R (1982) Mutations that affect membrane receptor for LDL are useful for studying normal receptor function. M J Physiol 43(1): E514.

6. Elina I (2008) Cellular cholesterol trafficking and compartmentalization. Nature Reviews Molecular Cell Biology 9(2): 125-138

7. Monty K (1999) Charting the fate of the "Good Cholesterol": Identification and characterization of the high-density lipoprotein receptor SR-BI Annu Rev Biochem 68: 523-558.

8. Silvia R, Fernando F, Roberto H, Meneghetti C, Brandizzi LI, et al. (2002) Uptake of a Cholesterol-Rich Emulsion by Breast Cancer. Gynecologic Oncology 85(3): 493-497.

9. Huang H, William C, Juan C, Zheng G (2015) Learning from Biology: Synthetic Lipoproteins for Drug Delivery. Wiley Interdiscip Rev Nanomed Nanobiotechnol 7(3): 298-314.

10. Didier M (2000) Rationale and applications of lipids as prodrug carriers European Journal of Pharmaceutical Sciences 11(Suppl 2): S15-S27.

11. Gamaleldin I, Fars K (2014) Low density lipoprotein bio nanoparticles: From cholesterol transport to delivery of anti-cancer drugs. Saudi Pharm J 22(6): 504-515.

12. Wim H, Paul A (2008) Drug delivery and nanoparticles: Applications and hazards. Int J Nanomedicine 3(2): 133-149.
13. Isachenko V, Lapidus I, Isachenko E, Krivokharchenko A, Kreienberg $R$, et al. (2009) Human ovarian tissue verification versus conventional freezing: morphological, endocrinological, and molecular biological evaluation. Reproduction 138(2): 319-327.

14. Hansen K, Knowlton S, Thyer C, Charleston JS, Soules MR, et al. (2008) A new model of reproductive aging: the decline in ovarian non-growing follicle number from birth to menopause. Hum Report 23(3): 699-708.

15. Oktay K, Oktem O (2008) Ovarian cryopreservation and transplantation for fertility preservation for medical indications: report of an ongoing experience. Fertil Steril 93(3): 762-768.

16. Piek M, Diest J, Verheijen H (2008) Ovarian carcinogenesis: an alternative hypothesis. Advances in Experimental Medicine and Biology 622: 79-87.

17. Shanmugaratnam K (1985) Prevention and Early Detection of Cancer. Cancer Detect Prev 8(4): 431-445.

18. Awwad A, Fares K (2014) Targeting cancer using cholesterol conjugates. Saudi Pharmaceutical Journal 22(1): 3-16.

19. Cariad C, Oliver D, Jonathan S, Holbrook K (2014) Immunotherapeutic approaches to ovarian cancer treatment. Immuno Therapy of Cancer 3:

20. Bell Mc K, Konner J, Tew W, Spriggs DR (2011) New drugs for ovarian cancer. Ann Oncol (Suppl 8): viii77-viii82.

21. Fischer J, Ganellin C (2006) Analogue-based Drug Discovery. John Wiley \& Sons, pp. 606.

22. Yunfei C, Hughes Fulford M (2001) Human prostate cancer cells lack feedback regulation of low-density lipoprotein receptor and its regulator, SREBP2. International Journal of cancer 91(1): 41-45.

23. Ian R, Gang Z (2007) Mimicking Nature's Nanocarrier: Synthetic Lowdensity Lipoprotein-like Nanoparticles for Cancer-drug Delivery. Nanomedicine 2(3)

24. Rensen C, Schiffelers R, Versluis A, Bijsterbosch MK, Kuijk Meuwissen ME, et al. (1997) Human recombinant apolipoprotein E-enriched liposomes can mimic low-density lipoproteins as carriers for the sitespecific delivery of antitumor agents. Mol Pharmacol 52(3): 445-55.

25. Timothy A, Craig P, Stan B (2009) Beyond chemotherapy: targeted therapies in ovarian cancer. Nature Reviews Cancer 9(3): 167-181.

26. Arnold I, Mayhew E (1986) Targeted drug delivery. Cancer 58(2 Suppl): 573-583.

27. Mills K, Needham D (1999) Targeted drug delivery. Expert opinion in therapeutic patents 9 (11): 1499-1513

28. Wadih A, Renata P, Erkki R (1998) Cancer Treatment by Targeted Drug Delivery to Tumor Vasculature in a Mouse Model. Science 279(5349): 377-380.

29. Laila Jaragh Alhadad (2018) In-vitro evaluation of HSP27 inhibitors function through HER2 pathway for ovarian cancer therapy. Transl Cancer Res 7(6): 1510-1517.

30. Laila J, Gamaleldin I, Fars K (2020) Design and encapsulation of anticancer dual HSP27 and HER2 inhibitor into low density lipoprotein to target ovarian cancer cells. Saudi Pharm J 28(4): 387-396. 


\section{ISSN: 2574-1241}

DOI: 10.26717/BJSTR.2021.35.005675

Laila Abdulmohsen Jaragh Alhadad. Biomed J Sci \& Tech Res

(C) (i) This work is licensed under Creative

Submission Link: https://biomedres.us/submit-manuscript.php

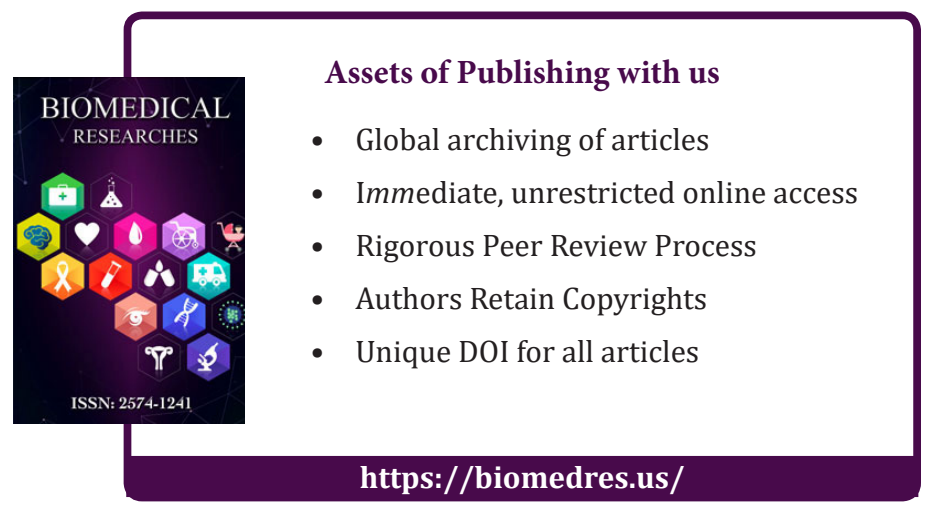

